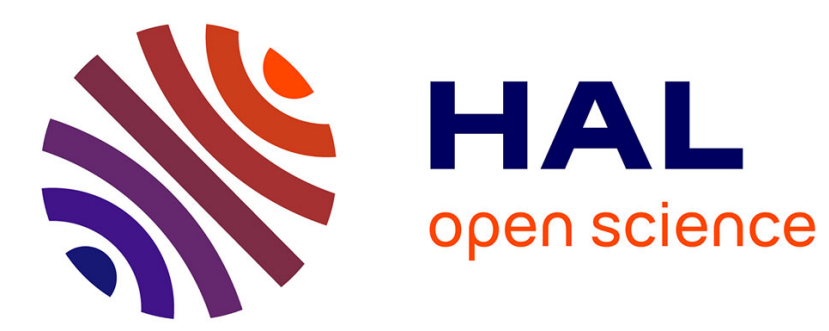

\title{
Efficient kinematics of a 2-1 and 3-1 CDPR with non-elastic sagging cables \\ Jean-Pierre Merlet
}

\section{To cite this version:}

Jean-Pierre Merlet. Efficient kinematics of a 2-1 and 3-1 CDPR with non-elastic sagging cables. CableCon 2021 - 5th International Conference on Cable-Driven Parallel Robots, Jul 2021, Virtual, France. 10.1007/978-3-030-75789-2_1. hal-03284195

\section{HAL Id: hal-03284195 \\ https://hal.inria.fr/hal-03284195}

Submitted on 12 Jul 2021

HAL is a multi-disciplinary open access archive for the deposit and dissemination of scientific research documents, whether they are published or not. The documents may come from teaching and research institutions in France or abroad, or from public or private research centers.
L'archive ouverte pluridisciplinaire HAL, est destinée au dépôt et à la diffusion de documents scientifiques de niveau recherche, publiés ou non, émanant des établissements d'enseignement et de recherche français ou étrangers, des laboratoires publics ou privés. 


\title{
Efficient kinematics of a 2-1 and 3-1 CDPR with non-elastic sagging cables
}

\author{
Jean-Pierre Merlet \\ HEPHAISTOS project, INRIA Sophia-Antipolis, France \\ Jean-Pierre.Merlet@inria.fr
}

\begin{abstract}
Solving the kinematics of CDPR is complex as soon as cable sagging is taken into account. We are considering here CDPRs having 2 cables whose extremities are attached at the same point on the platform (i.e. CDPRs allowing only translational motion). Regarding the cables we assume that they are nonelastic but have a mass so that they will exhibit sagging. We first show that the inverse and direct kinematics (IK and DK) amount to solve a square system of equations. We then show that these systems of equations may be reduced to solving an equation in a single variable that cannot be solved analytically but can easily be solved numerically. Using this result we show that the sagging will play a role on the result of the IK/FK only if the load mass is lower than a threshold. We then present some preliminary results regarding the case of 3 cables which is much more complex: the IK may be reduced to solving an equation in a single variable but this solving is numerically difficult. Here again it seems that sagging may be neglected if the load mass is high enough. We also present a preliminary analysis of the 3-1 case.
\end{abstract}

Keywords: cable-driven parallel robot,sagging cable, inverse kinematics,direct kinematics

\section{Introduction}

Solving the inverse kinematics and direct kinematics of CDPRs is an essential element for the design and control. Inverse kinematics is straightforward if the cable sagging is not taken into account but becomes complex as soon as sagging is considered with possibly several solutions if both the cable mass and its elasticity are taken into account [1]. Direct kinematics (DK) is even more complex whatever cable model is used. There are several variants of the DK problem:

1. finding the current platform pose using an estimation of the cable lengths but being given the platform pose a short time before the current one (this is often called real-time kinematics)

2. finding all solution poses based only on an estimation of the cable lengths

3. finding the current platform pose based on a combination of partial measurements of the pose components [2-5] and/or other information on the CDPR (e.g. cable angles [6-8] or force sensors [9]) and of the cable lengths 
We are considering that real-time kinematics is no more an issue as there are very efficient and certified algorithms (implementable in a distributed manner) that are able to solve it whatever the cable model is $[10,11]$. Many works have addressed point 3 but there are still improvements to be gained such as fusing additional measurements beside cable lengths and pose estimation (quite often obtained at a lower rate than the cable lengths) or mixing IK and DK to manage more efficiently slack cables [12,13]. Regarding point 2 the DK problem of CDPRs with ideal cable (no mass, no elasticity) has been addressed in several works[14-17] but there are much less works on the DK with sagging cables [18-22]. The proposed algorithms either cannot guarantee to find all solutions or are relatively expensive in term of computation time. This is mostly a consequence that sagging cable models are not algebraic so that classical methods such as elimination or Gröebner basis, which have been proven effective for classical parallel robots, cannot be used. In this paper we will address the inverse and direct kinematics of two specific classes of CDPR (figure 1):

- the planar CDPR 2-1 with 2 cables attached at the same point on the platform

- the spatial CDPR 3-1 with 3 cables attached at the same point on the platform

These CDPRs allow only for translational degrees-of-freedom and we will assume that the cables are non-elastic but have a mass. We will show that for both the 2-1 and
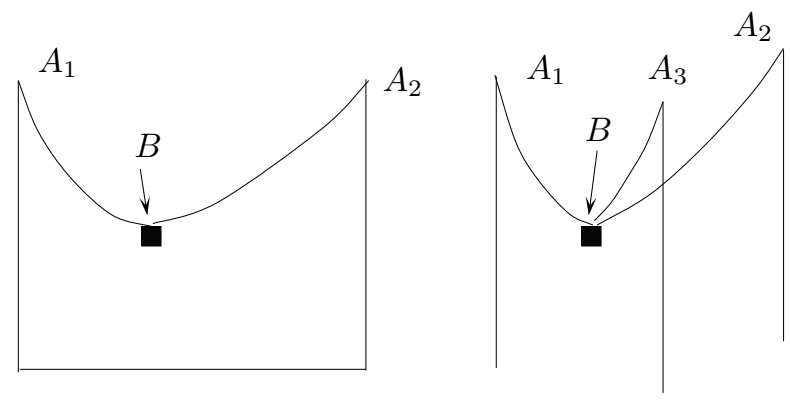

Fig. 1. The two CDPRs that will be studied: with 2 cables we have a planar motion and with 3 cables a spatial one.

3-1 CDPR the inverse kinematics may be reduced to solve a single equation in one unknown. This property will also be verified for the direct kinematics of the 2-1 CDPR.

\section{Cable model}

Beside the ideal and elastic-ideal mode, which assume that the cable shape is a line, multiple models that take into account elasticity and/or the cable mass have been proposed [23-27] but many of them have been simplified in order to be able to manage the kinematic issues. There is however a model usually called the Irvine model [28] that is 
extensively used in the analysis of cabled structures and it has been shown experimentally to be quite realistic for CDPRs [21]. This model is planar and takes into account both the elasticity and the mass of the cable. In this paper we will use a formulation of this model derived from [29] in which the elastic part is neglected. The motivation of neglecting elasticity is that the CDPRs we are considering are assumed to carry a relatively light load so that the cable tensions are relatively small.

We assume that a reference frame $\mathscr{R}=(0, \mathbf{x}, \mathbf{y}, \mathbf{z})$ has been defined with the $\mathbf{z}$ axis being the local vertical. The cables are attached at a common point $B$ on the platform whose coordinates in $\mathscr{R}$ are $\left(x_{b}, y_{b}, z_{b}\right)$. For a given cable we assume that the cable exits from the winch at a known fixed point $A$ with coordinates $\left(x_{a}, y_{a}, z_{a}\right)$ in $\mathscr{R}$. The platform exerts at $B$ a force $\mathbf{F}$ whose components in $\mathscr{R}$ are $\left(F_{x}, F_{y}, F_{z}\right)$. The cable tensions at $A, B$ will be respectively denoted $\tau_{A}, \tau_{B}, L_{0}$ is the cable length and $\mu$ is the linear density of the cable material, that is assumed to be identical for all cables. With this notation the cable model we will use is:

$$
\begin{aligned}
& x_{A}=x_{B}+\frac{F_{x}}{\mu g} \ln \left(\frac{\tau_{A}+F_{z}-\mu g L_{0}}{\tau_{B}+F_{z}}\right) \\
& y_{A}=y_{B}+\frac{F_{y}}{\mu g} \ln \left(\frac{\tau_{A}+F_{z}-\mu g L_{0}}{\tau_{B}+F_{z}}\right) \\
& z_{A}=z_{B}+\frac{\tau_{A}-\tau_{B}}{\mu g}
\end{aligned}
$$

We have also

$$
\tau_{B}^{2}=F_{x}^{2}+F_{y}^{2}+F_{z}^{2} \quad \tau_{A}^{2}=F_{x}^{2}+F_{y}^{2}+\left(F_{z}-\mu g L_{0}\right)^{2}
$$

In the sequel of this paper $F_{x}, F_{z}, L_{0}$ for cable $i$ will be denoted $F_{x_{i}}, F_{z_{i}}, L_{0_{i}}$.

\section{Analysis of the 2-1 CDPR}

The 2-1 CDPR is a planar robot with 2 cables and therefore 2 winch output points $A_{1}, A_{2}$. For the sake of simplicity we will assume that $A_{1}, A_{2}$ have the same height so that their coordinates are $(0,0),(d, 0)$. For the planar case we have to use only equations $(1,3,4)$ of the cable model.

\subsection{Inverse kinematics}

For the inverse kinematics we have to determine the cable lengths $L_{0_{1}}, L_{0_{2}}$ for given $x_{B}, z_{B}$. The unknowns of this problem are the $F_{x}, F_{z}, \tau_{A}, \tau_{B}, L_{0}$ for each cable so that we have a total of 10 unknowns. We have the set of equations $(1,3,4)$ for each cable which provide 8 constraints. At $B$ we have a load of mass $m$ and the mechanical equilibrium imposes the 2 constraints:

$$
F_{x_{1}}+F_{x_{2}}=0 \quad F_{z_{1}}+F_{z_{2}}=-m g
$$

so that we have a total of 10 constraints and hence the IK admits usually a finite number of solutions. Such a system may be solved with interval analysis but this method 
requires to have bounds for the unknowns but we have no systematic mean to determine bounds for the $F_{x}, F_{z}, L_{0}$. We define $U_{i}=e^{\mu g\left(x_{a_{i}}-x_{B}\right) / F_{x_{i}}}$ so that we have $U_{1}=e^{-x_{B} \mu g / F_{x_{1}}}$ and according to our notation we have $x_{B}>0, F_{x_{1}}>0$. As for $U_{2}$ taking into account that $F_{x_{2}}=-F_{x_{1}}$ we have $U_{2}=e^{-\left(d-x_{B}\right) \mu g / F_{x_{1}}}=e^{-\mu g d / F_{x_{1}}} / U 1$. We note that both $U_{1}$ and $U_{2}$ lies in the range $[0,1]$ and we have $F_{x_{1}}=-\mu g x_{B} / \ln \left(U_{1}\right)$. Equation (1) is written as

$$
\frac{\left(x_{a_{i}}-x_{b}\right) \mu g}{F_{x_{i}}}=\ln \left(\frac{\tau_{A_{i}}+F_{z_{i}}-\mu g L_{0_{i}}}{\tau_{B_{i}}+F_{z_{i}}}\right)
$$

Taking the exponential of both terms leads to

$$
\tau_{A_{i}}+F_{z_{i}}-\mu g L_{0_{i}}-U_{i}\left(\tau_{B_{i}}+F_{z_{i}}\right)=0
$$

while equation (3) is written as

$$
\mu g\left(z_{A_{i}}-z_{B}\right)-\left(\tau_{A_{i}}-\tau_{B_{i}}\right)=0
$$

These equations are linear in $\tau_{A_{i}}, \tau_{B_{i}}$ and by solving this system we obtain $\tau_{A_{i}}, \tau_{B_{i}}$ as functions of $L_{0_{i}}, F_{z_{i}}$ and $F_{x_{1}}$ as

$$
\tau_{A_{i}}=\frac{\mu g\left(\left(z_{A_{i}}-z_{b}\right) U_{i}-L_{0_{i}}\right)}{U_{i}-1}-F_{z_{i}} \quad \tau_{B_{i}}=\frac{\mu g\left(\left(z_{A_{i}}-z_{b}\right)-L_{0_{i}}\right)}{U_{i}-1}-F_{z_{i}}
$$

Reporting this result in (4) leads for each cable to 2 equations $F_{1}, F_{2}$ that are algebraic in $L_{0_{i}}$. Taking the resultant of these 2 equations leads to an equation whose unknowns are $U_{i}, F_{z_{i}}, F_{x_{1}}$. As $F_{z_{2}}=-m g-F_{z_{1}}$ we get for the 2 cables 2 equations $G_{1}, G_{2}$ whose unknowns are $U_{1}, U_{2}, F_{z_{1}}, F_{x_{1}}$. These equations are too large to be given here but they are written as

$$
G_{i}=a_{i}+b_{i} F_{x_{1}}^{2}+c_{i} F_{z_{1}}+d_{i} F_{z_{1}}^{2}
$$

where the coefficients $a_{i}, b_{i}, c_{i}, d_{i}$ are only functions of the $U_{i}$. These equations are algebraic in $F_{z_{1}}$ and their resultant $H$ in $F_{z_{1}}$ is an equation in $U_{1}, U_{2}, F_{x_{1}}$ only. As $U_{1}, U_{2}$ are functions of $F_{x_{1}}$ only, then $H$ is a function of the single unknown $F_{x_{1}}$. The equation $H=0$ may be written as

$$
H=\sum_{j=0}^{j=2} A_{j}\left(U_{1}, U_{2}\right) F_{x_{1}}^{2 j}
$$

As we have in $H$ both terms in $F_{x_{1}}^{j}$ and in $e^{A / F_{x_{1}}}$ there is no closed-form of the roots in $F_{x_{1}}$ of $H=0$. Note that using the expression of $F_{x_{1}}$ as function of $U_{1}$ we get an expression of $H$ in $U_{1}, U_{2}$ : a multi-Taylor expansion of $H$ at $1-\varepsilon$, for $U_{1}, U_{2}$, where $\varepsilon$ is supposed to be small, leads to $H>0$ meaning that there is a limit value $V$ for $F_{x_{1}}$ such that $H\left(\forall F_{x_{1}}>V\right)>0$ and therefore $V$ is an upper bound for the roots in $F_{x_{1}}$ of $H=0$. The value of $V$ may be safety set to $V=1000 x_{B} \mu g$ as in this case $U_{1}=0.999$. Interval analysis is then used to solve $H=0$ with a range for $F_{x_{1}}$ set to $[1 e-3, V]$. For each root $F_{x_{1}}$ of $H=0$ we calculate the common roots of $G_{1}=0, G_{2}=0$ which provide the possible value of $F_{z_{1}}$. For a pair $F_{x_{1}}, F_{z_{1}}$ the length(s) $L_{0_{i}}$ are obtained as the common root of $F_{1}=0, F_{2}=0$. 


\subsection{Example}

As example we consider the IK of a CDPR with $d=20 \mathrm{~m}$ for the pose $x_{B}=7 \mathrm{~m}, z_{B}=$ $-2 m$ with a load mass of $1 \mathrm{~kg}$ or $50 \mathrm{~kg}$. For this pose the distances $\rho_{1}, \rho_{2}$ between the $A, B$ points of cable 1, 2 are $7.280109 \mathrm{~m}$ and $13.15294 \mathrm{~m}$. The cables are made of Dyneema synthetic fiber with various cable diameter $d_{c}$ and figure 2 shows the values of $L_{0_{1}}, L_{0_{2}}$ and $\tau_{B_{1}}$ as a function of $d_{c}$ for a mass of $10 \mathrm{~kg}$ and of $50 \mathrm{~kg}$. A typical running time for the IK solver is about $3 \mathrm{~ms}$. As may be seen from the figure the differences between the
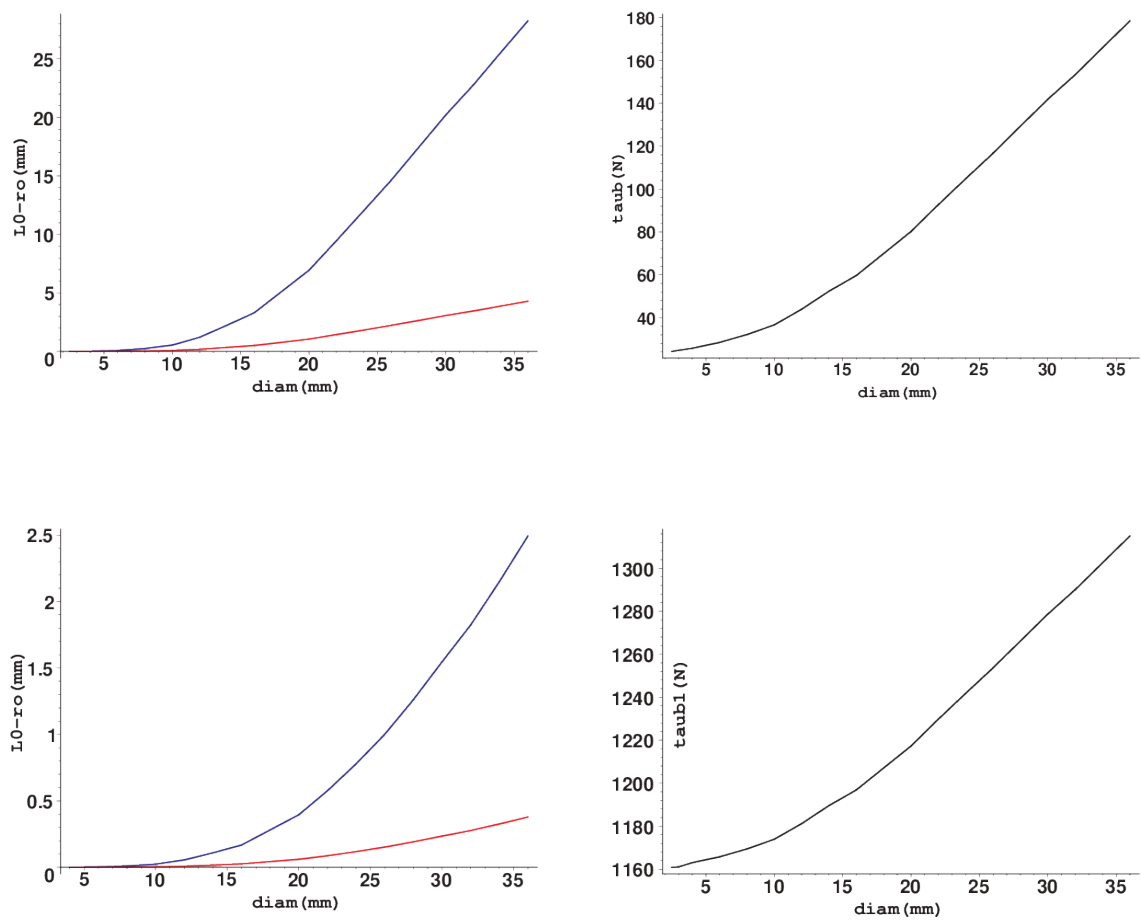

Fig. 2. For the IK the value of the ideal cable lengths are $\rho_{1}, \rho_{2}$ while with sagging the values are $L_{0_{1}}, L_{0_{2}}$. This figure presents the values of $L_{0_{1}}-\rho_{1}$ (red), $L_{0_{2}}-\rho_{2}$ (blue) and $\tau_{B_{1}}$ as a function of the cable diameter for a mass of $10 \mathrm{~kg}$ and of $50 \mathrm{~kg}$

ideal cable lengths is very small if $m=50 \mathrm{~kg}$ (in the worst case $0.38 \mathrm{~mm}$ and $2.5 \mathrm{~mm}$ for cable 1 and 2) while the tension at $B$ for cable 1 exhibits a more significant difference going from $1100 \mathrm{~N}$ to $1300 \mathrm{~N}$. If $m=10 \mathrm{~kg}$ there is a more significant difference in the $L_{0}\left(0.5 \mathrm{~cm}\right.$ for $L_{0_{1}}$ and $2.5 \mathrm{~cm}$ for $\left.L_{0_{2}}\right)$. There is also a significant change in the tension at $B$, going from $32 \mathrm{~N}$ to $180 \mathrm{~N}$. However being given the breaking point of the Dyneema cable a small diameter cable can be used in both cases with negligible effect 
on the kinematics solution compared to ideal cables. The influence of the load mass will be more extensively studied in the next section.

\subsection{Direct kinematics}

For this problem $L_{0_{1}}, L_{0_{2}}$ are given and we have to determine $x_{B}, z_{B}$. The solving process starts as for the IK by determining $\tau_{A_{i}}, \tau_{B_{i}}$ by solving equations $(1,3)$. We then report the result in the equations (4). Subtracting the left-side equation presented in (4) obtained for both cables and doing the same for the second equation leads to a system that is linear in $z_{B}, F_{z_{1}}$. This system is solved and the result is reported in the 2 equations (4) for the first cable. The unknowns in these equations are $F_{x_{1}}, U_{1}, U_{2}$ but as $U_{2}=e^{-d \mu g / F_{x_{1}}} / U_{1}=A / U_{1}$ we get 2 equations $D_{1}\left(F_{x_{1}}, U_{1}\right)=0, D_{2}\left(F_{x_{1}}, U_{1}\right)=0$ that are algebraic in $U_{1}$. The resultant of these equations in $U_{1}$ leads to the constraint $T\left(F_{x_{1}}\right)=0$. The function $T$ may be written as

$$
T=\sum_{j=0}^{j=12} u_{j} F_{x_{1}}^{j}
$$

where the coefficients $u_{j}$ are are algebraic function of $A$, which is restricted to lie in the range [0,1]. Furthermore we have $F_{x_{1}}=-d \mu g / \ln (A)$ so that function $T$ leads to a constraint $S(A)=0$. This equation is solved numerically to obtain the solution in $A$ which lead to the solution in $F_{x_{1}}$. Note that there is a symmetry in the $T$ constraint as $T\left(F_{x_{1}}, A\right)=T\left(-F_{x_{1}}, 1 / A\right)$.

For solving $S(A)=0$ we use interval analysis and the solutions provide the possible value of $F_{x_{1}}$. For a given $F_{x_{1}} D_{1}, D_{2}$ are algebraic function of $U_{1}$ only and their common roots are the possible values of $U_{1}$. Then $x_{B}$ is calculated as $x_{B}=F_{x_{1}} \ln \left(U_{1}\right) /(\mu g)$ while $F_{z_{1}}, z_{B}$ have been obtained in the process as functions of $A, U_{1}$. The total computation time is around $5 \mathrm{~ms}$ and may probably be reduced.

\subsection{Example}

We consider a CDPR with $d=20 \mathrm{~m}, \mu=0.023 \mathrm{~kg} / \mathrm{m}$ which correspond to a $6 \mathrm{~mm}$ Dyneema cable). For the cable lengths we use as cable lengths the distance between $A_{1}, A_{2}$ and the pose $x_{B}=7 m, z_{B}=-2 m$. We consider the following load masses: $0.1,1$ and $10 \mathrm{~kg}$ with the following differences between the calculated pose and $x_{B}, z_{B}$ in $\mathrm{cm}$ : (4.04, 18.95), (0.36, 1.595), (0.0053,0.023).

As analyzed in the IK and DK it appears that if the load mass is sufficiently large, then the sagging is negligible and the ideal cable models may be used. It is therefore of interest to determine the minimal load mass that will lead to a small difference $\Delta \mathbf{X}$ between the pose obtained with ideal cables and the one obtained with the sagging cables. Figure 3 presents the minimal mass that leads to a $\Delta \mathbf{X}=0.5 \mathrm{~cm}$ as function of the cable diameter. If the load has a larger mass than this minimum, then the sagging effect may be neglected. 

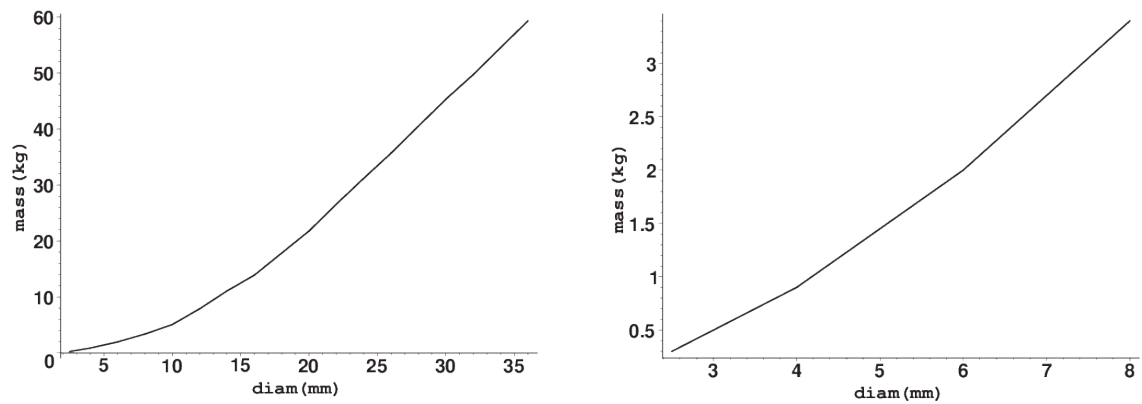

Fig. 3. We show here the load mass that leads to a distance of $5 \mathrm{~mm}$ between the 2-cables DK solution pose obtained for ideal cable and the one for sagging cable as function of the cable diameter If the load mass is larger than this mass, then the sagging may be neglected. On the right a detail for small diameter cables.

\section{Preliminary analysis of the 3-1 CDPR}

As will be seen in the next section the inverse kinematics of the 3-1 CDPR may also be reduced to solve a single equation, while we have not be able to obtain a similar result for the direct kinematics. Still we believe that the inverse kinematics process may be improved while reducing the complexity of the direct kinematics may be possible.

\subsection{Inverse kinematics}

Using equations $(1,2)$ we get $F_{y_{i}}=F_{x_{i}}\left(y a_{i}-y b\right) /\left(x a_{i}-x b\right)$. The mechanical equilibrium equations are $\sum_{j=1}^{j=3} F_{x_{i}}=\sum_{j=1}^{j=3} F_{y_{i}}=0$ and $\sum_{j=1}^{j=3} F_{z_{i}}=-m g$. The 2 first equations are linear in the $F_{x_{i}}$ so that we can get $F_{x_{2}}, F_{x_{3}}$ as functions of $F_{x_{1}}$. As in the 2-1 cable section we use the 2 equations (8) to get the value of the $\tau_{A_{i}}, \tau_{B_{i}}$. We then set $F_{z_{3}}=$ $-m g-F_{z_{1}}-F_{z_{2}}$ and consider for each cable the 2 equations $P_{1_{i}}, P_{2_{i}}$ of (4). For a given cable $j$ the unknowns in these 2 equations are $F_{x_{1}}, F_{z_{1}}, F_{z_{2}}, L_{0_{j}}, U_{j}$ and are algebraic in $L_{0_{j}}$. We compute the resultant $H_{j}$ of the 2 equations in $L_{0_{j}}$. For cable 2 and $3 H_{j}$ is algebraic in $F_{z_{2}}$ so that may calculate the resultant $R_{23}$ of $H_{2}, H_{3}$ in $F_{z_{2}}$. Finally we compute the resultant $W$ of $H_{1}, R_{23}$ in $F_{z_{1}}$ which is a function of $F_{x_{1}}, U_{1}, U_{2}, U_{3}$ where $U_{j}=e^{\mu g\left(x_{A_{i}}-x_{B}\right) / F_{x_{j}}}$. As $F_{x_{2}}, F_{x_{3}}$ has been obtained as function of $F_{x_{1}}$ the resultant $W$ is a function of $F_{x_{1}}$ may be written as

$$
W=\sum_{j=0}^{j=4} v_{j} F_{x_{1}}^{2 j}
$$

whose coefficients $v_{j}$ are functions of the $U_{j}$. If $W$ can be solved numerically for a given root of $W$ we get $F_{z_{1}}, F_{z_{2}}$ from $H_{1}, H_{2}$ which allow to calculate $F_{z_{3}}$. The 2 equations of 
(4) for each cable are now only algebraic functions of $L_{0_{j}}$ and their common roots are the possible values of $L_{0_{j}}$. However it appears that $W$ is difficult to solve as soon as the load mass is low.

\subsection{Direct kinematics}

Regarding the DK it has a single solution for ideal cable so that we can claim that it will also have a single solution for sagging cables as soon as the the DK equations are not singular [20]. Indeed the cable model is continuous in terms of $E, \mu$ with the deal cable being obtained for $E \rightarrow \infty, \mu=0$. Hence starting from the single solution obtained for the DK with ideal cable we may use a continuation scheme on $E, \mu$ to obtain the DK solution for given $E, \mu$. If the DK equations do not become singular during the continuation there will be a single solution. But if a singularity occurs, then the followed branch may split in several branches, thereby possibly leading to several DK solutions.

However we have not yet been able to reduce the DK system to a single variable equation. WE use an interval analysis based approach: bounds for $x_{B}, y_{B}, z_{B}$ are easily obtained and we consider very large intervals for $F_{x_{1}}, F_{z_{1}}, F_{z_{2}}, \tau_{A_{i}}, \tau_{B_{i}}$. The trick here is to assume that there is a single solution of the DK and to run a few iterations of the Newton scheme with as initial guess the center of the boxes obtained during the interval analysis algorithm and to stop the algorithm as soon as the Newton scheme converge. However the Newton scheme may converge to an incorrect solution. Indeed there is a symmetry in the DK equations so that if $\left(x_{B}, y_{B}, z_{B}, F_{x_{1}}, F_{z_{1}}, F_{z_{2}}, U_{i}, \tau_{A_{i}}, \tau_{B_{i}}\right)$ is a solution, then $\left(x_{B}, y_{B},-z_{B},-F_{x_{1}}, F_{z_{1}}, F_{z_{2}}, 1 / U_{i},-\tau_{A_{i}},-\tau_{B_{i}}\right)$ is also a solution: hence only solution(s) with positive $\tau_{A_{i}}, \tau_{B_{i}}$ have to be retained. Here again the sagging has an effect only for small load masses. For example we consider the CDPR with $x_{A_{1}}=y_{A_{1}}=$ $y_{A_{2}}=x_{A_{3}}=0, x_{A_{2}}=20, y_{A_{3}}=10$, the cable lengths equal to the distance from the $A_{i}$ to the pose $\mathscr{I}=(10,4,-3)$. For a load mass of $0 \mathrm{~kg}$ the pose will be $9.78,3.615,-2.56$ i.e. at a distance of $62.47 \mathrm{~cm}$ from $\mathscr{I}$. The difference between the reached pose and $\mathscr{I}$ decreases with the load mass: an error of $5 \mathrm{~mm}$ is obtained for a mass of $6.8 \mathrm{~kg}$ and an error of $1 \mathrm{~mm}$ for a load mass of $15.5 \mathrm{~kg}$. We have also investigated the convergence of the Newton scheme with as guess the DK solution obtained for ideal cables starting for various cable diameter and load mass. Curiously it appears that for cable diameter from $2.5 \mathrm{~mm}$ to $10 \mathrm{~mm}$ Newton diverges if the load mass is lower than $2 \mathrm{~kg}$ while this mass increases for larger cables ( $3 \mathrm{~kg}$ for a $12 \mathrm{~mm}$ diameter, $4.1 \mathrm{~kg}$ for $14 \mathrm{~mm}, 6.2 \mathrm{~kg}$ for $16 \mathrm{~mm}, 9.7 \mathrm{~kg}$ for $20 \mathrm{~mm}, 11.8 \mathrm{~kg}$ for $22 \mathrm{~mm}$ ). Hence for most existing prototypes the Newton scheme may be used to solve the DK.

\section{Conclusion}

The first contribution of this paper are very efficient FK and DK algorithms for CDPR with 2 sagging cables that are based on the solving of a function in one single variable. The second contribution is to show that the sagging of non-elastic synthetic fiber may be neglected as soon as the load mass is sufficiently large. At the opposite for a small mass the sagging may induce a very significant error in the CDPR positioning. The 
algorithms hence allows one to determine a lower bound $m_{m}$ for the load mass so that sagging may be neglected. Future work will address the case of elastic cables and it may be expected that the effect of elasticity will play a significant role only for a mass that is larger than a threshold $M_{M}$. If this is the case we will be able to determine a range $\left[m_{m}, m_{M}\right]$ for the load mass so that cable may be considered as ideal for the IK and DK, which greatly simplify the analysis.

Acknowledgment: the author want to thank the reviewers for their interesting comments.

\section{References}

1. Merlet J-P. A new generic approach for the inverse kinematics of cable-driven parallel robot with 6 deformable cables. In ARK, Grasse, June, 27-30, 2016.

2. Zavatta M. and others . A vision-based referencing procedure for cable-driven parallel manipulators. J. of Mechanisms and Robotics, 12(4), August 2020.

3. Korayem M.H. and others . A novel method for recording the position and orientation of the end effector of a spatial cable-suspended robot and using for closed-loop control. The International Journal of Advanced Manufacturing Technology, 72:739-755, 2014.

4. Mousavi M. and others . Sensory feedback performance improvement on RoboCab: an experimental approach to wiredriven parallel manipulator. In 4th International Conference on Robotics and Mechatronics, Teheran, October, 26-28, 2016.

5. Zi B., Lin J., and Quian S. Localization, obstacle avoidance planning and control of a cooperative cable parallel robot for multiple mobile cranes. Robotics and Computer-Integrated Manufacturing, 34:105-123, August 2015.

6. Fortin-Côté A., Cardou P., and Campeau-Lecours A. Improving cable driven parallel robot accuracy through angular position sensors. In IEEE Int. Conf. on Intelligent Robots and Systems (IROS), pages 4350-4355, Daejeon, October, 9-14, 2016.

7. Garant $X$. and others . Improving the forward kinematics of cable-driven parallel robots through cable angle sensors. In 3rd Int. Conf. on cable-driven parallel robots (CableCon), Québec, 2017.

8. Merlet J-P. Direct kinematics of CDPR with extra cable orientation sensors: the 2 and 3 cables case with perfect measurement and sagging cables. In IEEE Int. Conf. on Intelligent Robots and Systems (IROS), Vancouver, September, 24-28, 2017.

9. Oftadeh R., Aref M.M., and Taghirad H.D. Forward kinematic analysis of a planar cable driven redundant parallel manipulator using force sensors. In IEEE Int. Conf. on Intelligent Robots and Systems (IROS), pages 2295-2300, Taipei, October, 18-22, 2010.

10. Merlet J-P. On the real-time calculation of the forward kinematics of suspended cable-driven parallel robots. In 14th IFToMM World Congress on the Theory of Machines and Mechanisms, Taipei, October, 27-30, 2015.

11. Pott A. An algorithm for real-time forward kinematics of cable-driven parallel robots. In ARK, pages 529-538, Piran, June 28- July 1, 2010.

12. Zake Z. and others . Vision-based control and stability analysis of a cable-driven parallel robot. IEEE Robotics and Automation Letters, 4(2), April 2019.

13. Merlet J-P., Papegay Y., and Gasc A-V. The Princes tears, a large cable-driven parallel robot for an artistic exhibition. In IEEE Int. Conf. on Robotics and Automation, Paris, May 31August 31, 2020.

14. Abbasnejad G. and Carricato M. Direct geometrico-static problem of underconstrained cable-driven parallel robots with n cables. IEEE Trans. on Robotics, 31(2):468-478, April 2015. 
15. Berti A., Merlet J-P., and Carricato M. Solving the direct geometrico-static problem of underconstrained cable-driven parallel robots by interval analysis. Int. J. of Robotics Research, 35(6):723-739, 2016.

16. Husty M. and Zsombor-Murray P. Geometric contribution to the analysis of 2-2 wire driven cranes. In Int. Conf. on Interdisciplinary Applications of Kinematics, pages 1-8, Lima, September, 9-11, 2013.

17. Pott A. and Tempel P. A unified approach to forward kinematics for cable-driven parallel robots based on energy. In ARK, Bologna, July, 1-5, 2018.

18. Gouttefarde M., Nguyen D.Q., and Baradat C. Kinetostatics analysis of cable-driven parallel robots with consideration of sagging and pulleys. In ARK, pages 213-221, Ljulbjana, June 29- July 3, 2014.

19. Merlet J-P. The forward kinematics of cable-driven parallel robots with sagging cables. In 2nd Int. Conf. on cable-driven parallel robots (CableCon), pages 3-16, Duisburg, August, 24-27, 2014.

20. Merlet J-P. A generic numerical continuation scheme for solving the direct kinematics of cable-driven parallel robot with deformable cables. In IEEE Int. Conf. on Intelligent Robots and Systems (IROS), Daejeon, October, 9-14, 2016.

21. Riehl N. and others . Effects of non-negligible cable mass on the static behavior of large workspace cable-driven parallel mechanisms. In IEEE Int. Conf. on Robotics and Automation, pages 2193-2198, Kobe, May, 14-16, 2009.

22. Such M. and others. An approach based on the catenary equation to deal with static analysis of three dimensional cable structures. Engineering structures, 31(9):2162-2170, 2009.

23. Baklouti S., Caro S., and Courteille E. Dynamic and oscillatory motions of cable-driven parallel robots based on a non-linear cable tension model. J. of Mechanisms and Robotics, 9, September 2017.

24. Ferravante V. and others . Dynamic analysis of high precision construction cable-driven parallel robots. Mechanism and Machine Theory, 135:54-64, 2019.

25. Miermeister P. and others . An elastic cable model for cable-driven parallel robots including hysteresis effect. In 2nd Int. Conf. on cable-driven parallel robots (CableCon), pages 17-28, Duisburg, August, 24-27, 2014.

26. Miyasaka M. and others . Hysteresis model of longitudinally loaded cable for cable driven robots and identification of the parameters. In IEEE Int. Conf. on Robotics and Automation, Stockholm, May, 16-20, 2016.

27. Piao J. and others . Open-loop position control of a polymer cable-driven parallel robot via a viscoelastic cable model for high payload workspaces. Advances in Mechanical Engineering, 9(12), 2017.

28. Irvine H. M. Cable Structures. MIT Press, 1981.

29. Nguyen D.Q. and Gouttefarde M. Stiffness matrix of 6-dof cabl-driven parallel robots and its homogenization. In $A R K$, Ljulbjana, June 29- July 3, 2014. 